\title{
Lack of association between CYP1A1 T6235C polymorphism and coronary artery disease: Evidence from a meta-analysis
}

\author{
JIN ZHANG $^{1 *}$, YONGWEI WANG ${ }^{2 *}$, YUANLIN LIU $^{3}$ and XIAOZHONG ZHANG ${ }^{1}$ \\ ${ }^{1}$ Department of Cardiology, The Affiliated Hospital of Academy of Military Medical Sciences, Beijing 100071; \\ ${ }^{2}$ Department of Emergency, People's Liberation Army General Hospital, Beijing 100853; \\ ${ }^{3}$ Institute of Basic Medical Sciences, Academy of Military Medical Sciences, Beijing 100850, P.R. China
}

Received July 31, 2012; Accepted November 12, 2012

DOI: $10.3892 / \mathrm{mmr} .2012 .1212$

\begin{abstract}
A number of studies have evaluated the correlation between the cytochrome P450 1A1 (CYP1A1) T6235C polymorphism and coronary artery disease (CAD) risk, however, at present the results remain inconclusive. To provide a more robust investigation of this correlation, a meta-analysis was performed. In the present study, a systematic search of PubMed, Embase and CBM databases for studies published prior to June 6, 2012 was performed. The correlation between the CYP1A1 T6235C polymorphism and CAD risk was assessed by calculating pooled odds ratios (ORs) and $95 \%$ confidence intervals (95\% CIs). Seven studies with a total of 2,903 cases and 2,304 controls were included in the meta-analysis. Overall, the CYP1A1 T6235C polymorphism was not correlated with $\mathrm{CAD}$ risk $(\mathrm{C}$ vs. $\mathrm{T}$ : $\mathrm{OR}=1.03$; 95\% CI, 0.87-1.22; $\mathrm{P}=0.728$; CC vs. TT: OR=1.04; 95\% CI, 0.84-1.19; $\mathrm{P}=0.699 ; \mathrm{CC}+\mathrm{TC}$ vs. TT: $\mathrm{OR}=1.04 ; 95 \% \mathrm{CI}, 0.93$ 1.18; $\mathrm{P}=0.478$; $\mathrm{CC}$ vs. TC+TT: $\mathrm{OR}=1.04 ; 95 \% \mathrm{CI}, 0.85-1.28$; $\mathrm{P}=0.704)$. A meta-analysis of five high-quality studies demonstrated that the CYP1A1 T6235C polymorphism is not correlated with risk of CAD in 4 genetic models. Ethnic subgroup analyses identified no significant correlation in Caucasian, Asian and African populations. The present meta-analysis study indicates that the CYP1A1 T6235C polymorphism is not correlated with CAD risk. Additional studies with a larger sample size and consistent design must be performed to confirm the present hypothesis.
\end{abstract}

Correspondence to: Professor Xiaozhong Zhang, Department of Cardiology, Affiliated Hospital of Academy of Military Medical Sciences, 8 Dongda Street, Beijing 100071, P.R. China

E-mail: xiaozhongz357@yahoo.com.cn

*Contributed equally

Key words: CYP1A1 polymorphism, coronary artery disease, meta-analysis

\section{Introduction}

Coronary artery disease (CAD), including its most severe complication, myocardial infarction, is the leading cause of mortality worldwide $(1,2)$. There are several traditional risk factors of CAD, including hypertension, diabetes mellitus, dyslipidemia and smoking, however, these factors explain only two-thirds of the observed clinical events $(3,4)$. In addition, not all individuals exposed to those risk factors develop CAD, which is indicative of other causes, including genetic susceptibility, which may contribute to variations in host susceptibility to CAD. Identification of CAD susceptibility genes highlights the link between CAD and inflammation and immunity, as well as the biological insights that may be gained from a genetic understanding of CAD $(5,6)$.

Environmental pollutants, including polycyclic aromatic hydrocarbons (PAHs), aldehydes and metals, contribute to the incidence, severity and risk of CAD by affecting atherogenesis, thrombosis and blood pressure. Cytochrome P450 1A1 (CYP1A1) is a member of the super family of cytochrome P450 enzymes important for detoxification of PAHs $(7,8)$. The CYP1A1 gene codes for a phase I enzyme associated with detoxification pathways that protect against damage caused by reactive metabolites of a number of chemicals, including steroids. Numerous mutations in CYP1A1 have been described and a $\mathrm{T} \rightarrow \mathrm{C}$ mutation in the non-coding 3'-flanking region of the gene (MspI, T6235C polymorphism, rs4646903) is the most commonly studied polymorphism (9). CYP1A1 T6235C in the 3'-flanking region, is associated with increased transcript half-life and therefore increased enzyme activity leading to elevated levels of activated metabolites $(9,10)$. The CYP1A1 T6235C polymorphism is one of the most extensively studied genes for susceptibility to various diseases over the last two decades. A number of studies have investigated the correlation between the CYP1A1 T6235C polymorphism and CAD risk, however, the results remain inconclusive (11-16). Primarily, single studies have been performed to investigate the correlation between the CYP1A1 T6235C polymorphism and CAD risk. In the present study, a meta-analysis was performed to increase the statistical power to examine the correlation between the CYP1A1 T6235C polymorphism and CAD risk. The meta-analysis of observational studies in epidemiology (MOOSE) consensus statement was followed 
during stages of design, implementation and reporting of this meta-analysis (17).

\section{Materials and methods}

Search strategy. A comprehensive search of PubMed, Embase and the Chinese Biomedical Database (CBM) databases prior to June 6, 2012 was performed. Search terms for the CYP1A1 T6235C polymorphism and CAD risk were combined and included 'cytochrome P4501A1', 'CYP1A1', 'T6235C', 'rs4646903' or 'MspI'; and 'coronary artery disease', 'coronary heart disease' or 'myocardial infarction'. There was no language limitation. Review articles and bibliographies of relevant literature were manually scanned to identify additional eligible studies.

Study eligibility. The criteria used for the study selection were: i) Evaluation of the correlation between the CYP1A1 T6235C polymorphism and CAD risk; ii) studies with case-control design; iii) studies with full text articles; and iv) sufficient data for estimating an odds ratio (OR) with its corresponding 95\% confidence interval (CI). Studies investigating progression, severity, phenotype modification, response to treatment or survival were excluded from the present meta-analysis. Family-based association studies were also excluded due to utilization of various study designs. In cases where multiple articles publishing data on the same population were identified, the publication with the most complete data set was included.

Data extraction. Two investigators independently extracted data using a standardized data extraction form. Discrepancies were resolved by discussion and if consensus was not achieved the decision was made by a third investigator. The information sought from each publication included author, year of publication, source of controls, country of origin, ethnicity of participants, adjustment for known confounding variables and genotype information. In studies of multiple ethnic groups, data were extracted separately for each ethnic group. Ethnicity of the participants was categorized as Caucasian, Asian and Africans.

Quality assessment. Quality assessment for case-control studies was assessed using the Newcastle Ottawa scale (NOS) as recommended by the Cochrane Non-Randomized Studies Methods Working Group (18-20). This instrument was developed to assess the quality of non-randomized studies, specifically cohort and case-control. Based on the NOS, case-control studies were judged based on three broad perspectives: selection of study groups (4 criteria), comparability of study groups (1 criteria) and ascertainment of outcome of interest ( 3 criteria). Considering the variability in quality of observational studies identified on our initial literature search, we considered studies that met $\geq 5$ of the NOS criteria as high quality (18-20). Hardy-Weinberg Equilibrium (HWE) served as a surrogate to assess study quality. The effect of HWE was associated with problems in the design and conduct of genetic association studies and therefore studies with departures from HWE were judged as low quality (21).

Statistical analysis. For assessment of deviation from HWE in the reported genotype frequencies among controls, the appro- priate goodness-of-fit $\chi^{2}$ test was carried out (21). Pooled ORs and $95 \%$ CIs were performed to assess the strength of the correlation between the CYP1A1 T6235C polymorphism and CAD risk. Pooled ORs with corresponding 95\% CIs for all studies were calculated and subgroup analyses were then performed in the ethnic groups (Caucasians, Asians and Africans). Pooled ORs were performed for the allele (C vs. T), homozygous (CC vs. TT), dominant (CC+CT vs. TT) and recessive models (CC vs. $\mathrm{CT}+\mathrm{TT})$. Statistical heterogeneity among studies was estimated with $\mathrm{Q}$ and $\mathrm{I}^{2}$ statistics $(22,23)$. A P-value for the Q statistic $>0.10$ or $\mathrm{I}^{2}$ value $<50 \%$ indicated a lack of marked heterogeneity among the studies. On the basis of heterogeneity test results, the fixed-effects (Mantel-Haenszel) or random-effects model (DerSimonian and Laird) was selected to calculate pooled OR $(24,25)$. Potential publication bias was investigated using the Begg's funnel plot and an asymmetric plot indicated possible publication bias. Funnel-plot asymmetry was further determined by the Egger's linear regression test method (26). Stata Version 12 (Stata Corp, College Station, TX, USA) was used for statistical analyses. Two-sided $\mathrm{P}<0.05$ was considered to indicate a statistically significant difference for all analyses.

\section{Results}

Characteristics of included studies. The search criteria identified 38 abstracts. Following elimination of studies that did not meet the criteria and exclusion of 19 records, 9 full-text publications were preliminarily identified for further evaluation (11-16,27-29). Each original manuscript was reviewed and data were extracted. Two publications were excluded, including a case-only study(27) and a study with limited data (28). Therefore, 7 studies with a total of 2,903 CAD cases and 2,304 controls were included in the meta-analysis (11-16,29). All 7 studies were hospital-based case-control studies. There were 4 studies on Caucasian populations $(11,12,14,15), 2$ on Asians $(16,29)$ and 1 on Africans (13). The number of cases varied between 114 and 873 and controls varied between 53 and 932. The CYP1A1 T6235C genotype distribution in the control groups were all consistent with HWE, with the exception of 1 study (29). According to the quality criteria, there were 5 studies with high quality (12-16). The remaining studies were considered low quality $(11,29)$.

Quantitative synthesis. Pooled ORs and the corresponding 95\% CIs are shown in Table I. Overall, the CYP1A1 T6235C polymorphism was not correlated with CAD risk (C vs. T: $\mathrm{OR}=1.03 ; 95 \% \mathrm{CI}, 0.87-1.22 ; \mathrm{P}=0.728 ; \mathrm{CC}$ vs. TT: $\mathrm{OR}=1.04$; 95\% CI, 0.84-1.29; $\mathrm{P}=0.699 ; \mathrm{CC}+\mathrm{TC}$ vs. TT: $\mathrm{OR}=1.04$, 95\% CI, 0.93-1.18; $\mathrm{P}=0.478 ; \mathrm{CC}$ vs. TC+TT: $\mathrm{OR}=1.04$, 95\% CI, 0.85-1.28; P=0.704; Figs. 1-4). Meta-analyses of the 5 high-quality studies demonstrated that the CYP1A1 T6235C polymorphism was not correlated with risk of CAD in 4 genetic models (Table I). Ethnic subgroup analyses revealed no significant correlation was detected in Caucasians, Asians and Africans (Table I).

Publication bias. Begg's funnel plot and Egger's test were performed to assess publication bias. The shapes of the funnel plots did not reveal any evidence of marked asymmetry in all comparison models. Egger's test was further 
Table I. Meta-analysis of the correlation between the CYP1A1 T6235C polymorphism and CAD risk.

\begin{tabular}{|c|c|c|c|c|c|}
\hline Studies & Contrast model & Participants $^{\mathrm{a}}$ & OR $(95 \% \mathrm{CI})$ & $\mathrm{P}_{\mathrm{OR}}$ & $\mathrm{I}^{2}(\%)$ \\
\hline \multirow[t]{4}{*}{ Total } & C vs. T & $6(4511)$ & $1.03(0.87-1.22)$ & 0.728 & 55.8 \\
\hline & CC vs. TT & $6(4511)$ & $1.04(0.84-1.29)$ & 0.699 & 46.6 \\
\hline & CC/CT vs. TT & $7(5207)$ & $1.04(0.93-1.18)$ & 0.478 & 23.4 \\
\hline & $\mathrm{CC}$ vs. CT/TT & $6(4511)$ & $1.04(0.85-1.28)$ & 0.704 & 38.1 \\
\hline \multirow[t]{4}{*}{ High quality } & C vs. T & $5(3758)$ & $0.98(0.88-1.09)$ & 0.698 & 48.6 \\
\hline & CC vs. TT & $5(3758)$ & $0.92(0.71-1.18)$ & 0.513 & 30.8 \\
\hline & CC/CT vs. TT & $5(3758)$ & $0.99(0.86-1.13)$ & 0.861 & 23.5 \\
\hline & $\mathrm{CC}$ vs. CT/TT & $5(3758)$ & $0.93(0.73-1.19)$ & 0.573 & 24.4 \\
\hline \multirow[t]{4}{*}{ Caucasian } & C vs. T & $3(2286)$ & $0.94(0.58-1.52)$ & 0.792 & 72.3 \\
\hline & CC vs. TT & $3(2286)$ & $0.82(0.31-2.13)$ & 0.680 & 54.6 \\
\hline & CC/CT vs. TT & $4(2982)$ & $1.02(0.73-1.41)$ & 0.919 & 50.5 \\
\hline & $\mathrm{CC}$ vs. CT/TT & $3(2286)$ & $0.91(0.69-1.21)$ & 0.526 & 48.2 \\
\hline \multirow[t]{4}{*}{ Asian } & C vs. T & $2(1442)$ & $1.14(0.98-1.34)$ & 0.099 & 0.0 \\
\hline & CC vs. TT & $2(1442)$ & $1.33(0.95-1.84)$ & 0.093 & 0.0 \\
\hline & CC/CT vs. TT & $2(1442)$ & $1.14(0.92-1.42)$ & 0.234 & 0.0 \\
\hline & $\mathrm{CC}$ vs. CT/TT & $2(1442)$ & $1.27(0.93-1.73)$ & 0.127 & 0.0 \\
\hline \multirow[t]{4}{*}{ African } & C vs. T & $1(783)$ & $1.02(0.75-1.38)$ & 0.897 & NA \\
\hline & CC vs. TT & $1(783)$ & $0.53(0.16-1.79)$ & 0.310 & NA \\
\hline & CC/CT vs. TT & $1(783)$ & $1.08(0.78-1.51)$ & 0.648 & NA \\
\hline & $\mathrm{CC}$ vs. CT/TT & $1(783)$ & $0.52(0.16-1.74)$ & 0.289 & NA \\
\hline
\end{tabular}

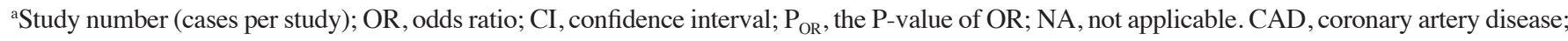
CYP1A1, cytochrome P450 1A1.

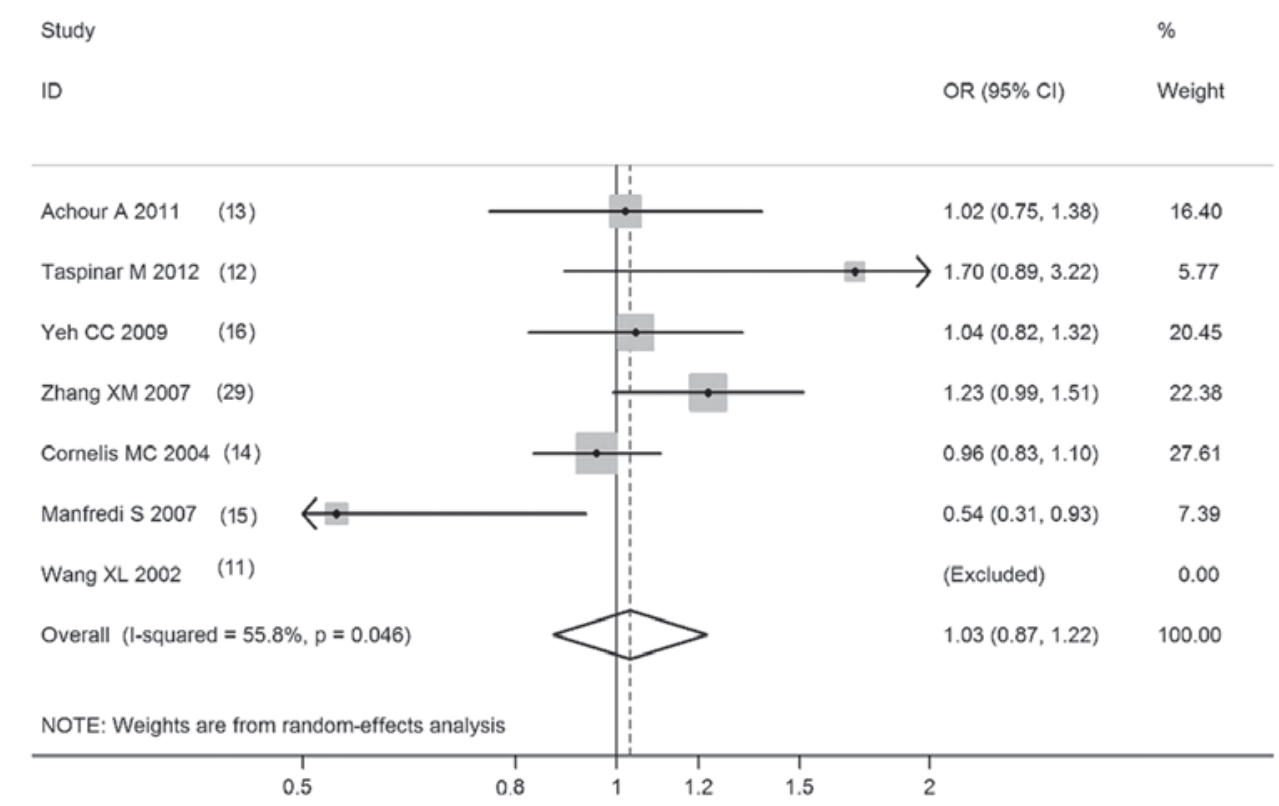

Figure 1. Meta-analysis of the correlation between the CYP1A1 T6235C polymorphism and CAD risk (C vs. T). CYP1A1, cytochrome P450 1A1; CAD, coronary artery disease; OR, odds ratio; CI, confidence interval.

used to provide statistical evidence of funnel plot asymmetry and the results were consistent with no evidence of publication bias (Egger's test values: $\mathrm{C}$ vs. T: $\mathrm{P}=0.690$; $\mathrm{CC}$ vs. TT:
$\mathrm{P}=0.661 ; \mathrm{CC} / \mathrm{TC}$ vs. TT: $\mathrm{P}=0.450 ; \mathrm{CC}$ vs. TC/TT: $\mathrm{P}=0.693)$. Therefore, a low risk of publication bias was detected in the present meta-analysis. 


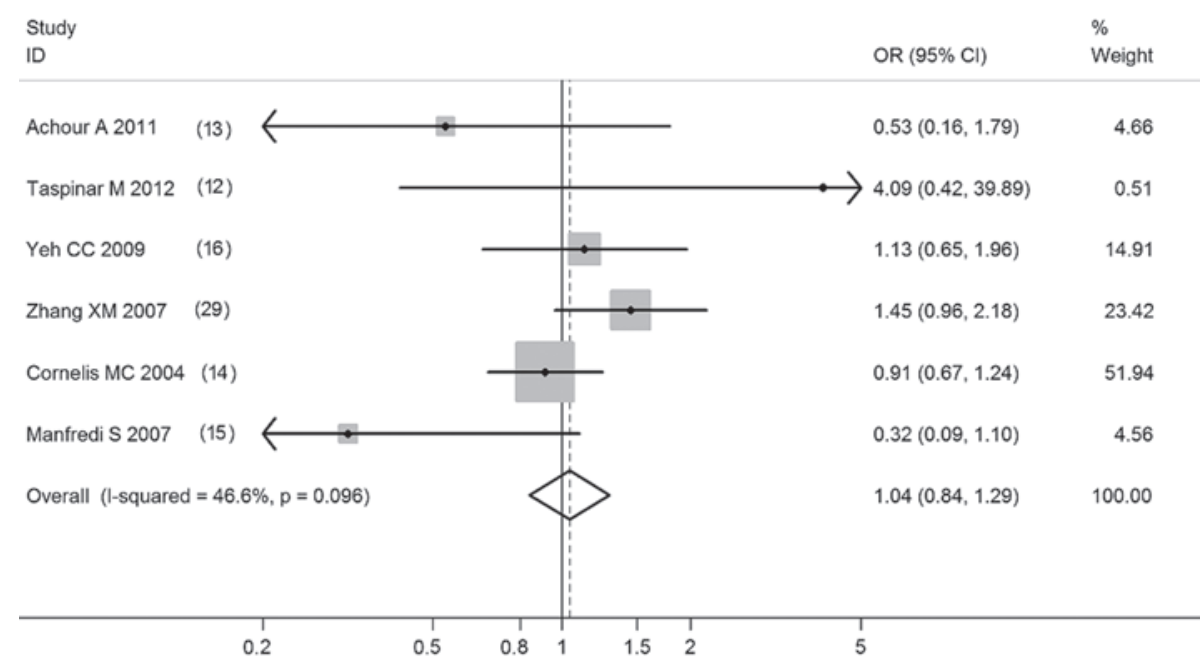

Figure 2. Meta-analysis of the correlation between the CYP1A1 T6235C polymorphism and CAD risk (CC vs. TT). CYP1A1, cytochrome P450 1A1; CAD, coronary artery disease; OR, odds ratio; CI, confidence interval.

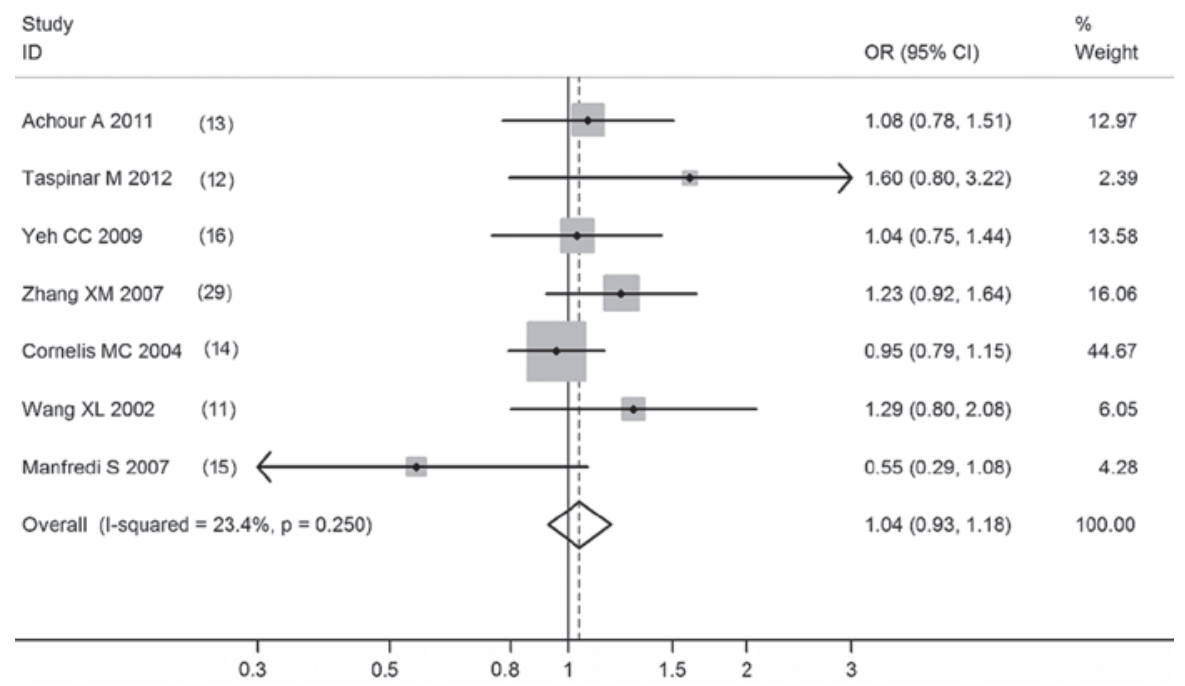

Figure 3. Meta-analysis of the correlation between the CYP1A1 T6235C polymorphism and CAD risk (CC/CT vs. TT). CYP1A1, cytochrome P450 1A1; $\mathrm{CAD}$, coronary artery disease; OR, odds ratio; CI, confidence interval.

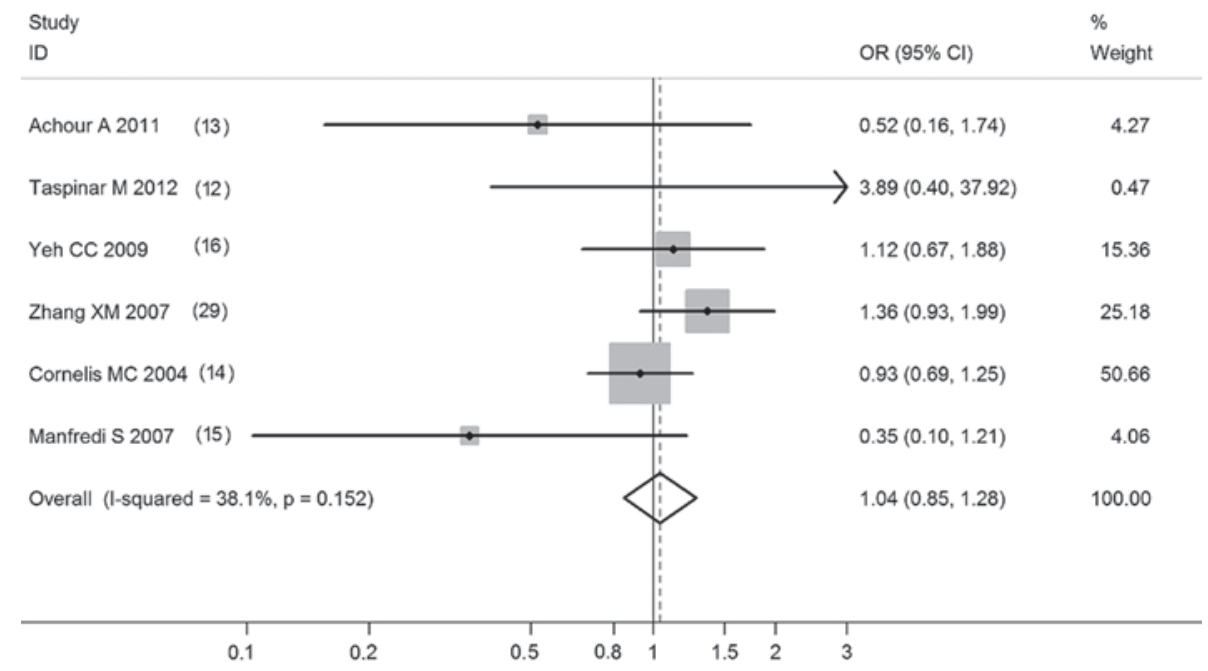

Figure 4. Meta-analysis of the correlation between the CYP1A1 T6235C polymorphism and CAD risk (CC vs. TT/CT). CYP1A1, cytochrome P450 1A1; $\mathrm{CAD}$, coronary artery disease; OR, odds ratio; CI, confidence interval. 


\section{Discussion}

CYP1A1 is a phase I extrahepatic metabolic enzyme involved in the bioactivation of carcinogenic PAHs, including benzopyrene. PAHs present in smoked foods, tobacco smoke and ubiquitous in urban environments in large cities are considered to be responsible for an elevated risk of specific types of cancer and cardiovascular diseases. Considering the importance of CYP1A1, it is biologically plausible that CYP1A1 polymorphisms may modulate the risk of cancer and it is well documented that CYP1A1 is important in arachidonic acid metabolism. The metabolites generated by the process are associated with cardiovascular physiology $(16,30,31)$. In addition, CYP1A1 is involved in the metabolic activation of tobacco-derived PAHs, molecules associated with carcinogenesis and atherosclerosis. A number of CYP1A1 polymorphisms increase transcript half-life, leading to increased enzyme activity and a subsequent elevation in levels of activated metabolites $(9,10)$. Therefore, a correlation between CYP1A1 polymorphisms and CAD risk is possible.

A number of previous studies have evaluated the correlation between the CYP1A1 T6235C polymorphism and CAD risk, however, the effect of this polymorphism on CAD remains inconclusive. Previously, small sample genetic association studies were performed. These studies had various designs and methodologies and insufficient power, qualities associated with an increased risk of false results. Combining data from all eligible studies by meta-analysis reduces random error and increases the accuracy of genetic association data. To provide a more robust estimate of the hypothesized correlation, a meta-analysis was performed. In the present study, a comprehensive meta-analysis of literature on the correlation between the CYP1A1 T6235C polymorphism and CAD risk was performed. On the basis of our inclusion criteria, 7 studies with a total of 2,903 cases and 2,304 controls were included in the meta-analysis. Overall, the CYP1A1 T6235C polymorphism was not correlated with CAD risk (C vs. T: $\mathrm{OR}=1.03 ; 95 \% \mathrm{CI}, 0.87-1.22 ; \mathrm{P}=0.728$; CC vs. TT: $\mathrm{OR}=1.04 ; 95 \% \mathrm{CI}, 0.84-1.29 ; \mathrm{P}=0.699 ; \mathrm{CC}+\mathrm{TC}$ vs. TT: $\mathrm{OR}=1.04 ; 95 \% \mathrm{CI}, 0.93-1.18 ; \mathrm{P}=0.478 ; \mathrm{CC}$ vs. TC+TT: $\mathrm{OR}=1.04 ; 95 \% \mathrm{CI}, 0.85-1.28 ; \mathrm{P}=0.704)$. Meta-analyses of five high-quality studies demonstrated that the CYP1A1 T6235C polymorphism was not correlated with risk of CAD in four genetic models. Ethnic subgroup analyses revealed that no significant correlation was identified in Caucasian, Asian and African populations. Therefore, this meta-analysis indicates that the CYP1A1 T6235C polymorphism is not correlated with CAD risk.

Four polymorphisms, including T6235C (a substitution in the $3^{\prime}$ non-coding region), A2455G (isoleucine to valine transition at codon 462), $\mathrm{T} 3205 \mathrm{C}$ (a transition mutation in the 3 ' non-coding region) and $\mathrm{C} 2453 \mathrm{~A}$ (threonine to asparagine transition at codon 461) were previously identified in the CYP1A1 gene (32). C2453A is extremely rare and T3205C exists only in African and African-American individuals. Therefore, the majority of studies have focused on the CYP1A1 T6235C and CYP1A1 A2455G polymorphisms (32). The CYP1A1 A2455G polymorphism was previously found to be correlated with risk of several common types of cancer, however, the CYP1A1 T6235C polymorphism is not correlated with this risk (33-35). These outcomes indicate that the CYP1A1 A2455G polymorphism may have additional effects on the CYP1A1 enzyme compared with the CYP1A1 T6235C polymorphism. Previous studies have concentrated on the correlation between the CYP1A1 T6235C polymorphism and CAD risk, however, limited studies have analyzed this correlation with respect to the CYP1A1 A2455G polymorphism. Therefore, additional studies are required to assess this correlation.

The present study is associated with several limitations that must be considered when interpreting the results. Firstly, inclusion of only seven studies with relatively small sample sizes and poor validation was the main limitation of the metaanalysis. In addition, only two studies on Asian and four on Caucasian populations were included in the analysis. Two methods were utilized to assess publication bias, however, these methods may be associated with low power for the detection of publication bias risk in cases of limited study numbers. Therefore, additional studies of larger sample size and consistent design are required for comprehensive analysis of this correlation. Secondly, the main analysis was based on unadjusted estimates due to lack of adjusted estimates. A more precise analysis is achieved when adjusted estimates are available for all studies (36). Considerable variability in study design and control selection was revealed in the present metaanalysis. To obtain results from meta-analysis of homogeneous studies, additional studies with adjusted estimates are required. Thirdly, gene-environmental factor interactions were not fully addressed due to insufficient data. Future studies must further assess the possible gene-environmental interactions, including gene-smoking interactions, in the correlation between the CYP1A1 T6235C polymorphism and CAD risk.

In conclusion, the present study has demonstrated that the CYP1A1 T6235C polymorphism is not correlated with CAD risk. Additional studies with larger sample size and consistent design must be performed to confirm this finding.

\section{References}

1. White HD and Chew DP: Acute myocardial infarction. Lancet 372: 570-584, 2008

2. Pfisterer ME, Zellweger MJ and Gersh BJ: Management of stable coronary artery disease. Lancet 375: 763-772, 2010.

3. Hansson GK: Inflammation, atherosclerosis and coronary artery disease. N Engl J Med 352: 1685-1695, 2005.

4. Libby $\mathrm{P}$ and Theroux P: Pathophysiology of coronary artery disease. Circulation 111: 3481-3488, 2005.

5. Samani NJ, Erdmann J, Hall AS, et al: Genomewide association analysis of coronary artery disease. N Engl J Med 357: 443-453, 2007.

6. Topol EJ, Smith J, Plow EF and Wang QK: Genetic susceptibility to myocardial infarction and coronary artery disease. Hum Mol Genet 15: R117-R123, 2006.

7. Nebert DW and Gonzalez FJ: P450 genes: structure, evolution and regulation. Annu Rev Biochem 56: 945-993, 1987.

8. Whitlock JP Jr: Induction of cytochrome P4501A1. Annu Rev Pharmacol Toxicol 39: 103-125, 1999.

9. Sivaraman L, Leatham MP, Yee J, Wilkens LR, Lau AF and Le Marchand L: CYP1A1 genetic polymorphisms and in situ colorectal cancer. Cancer Res 54: 3692-3695, 1994.

10. Landi MT, Bertazzi PA, Shields PG, et al: Association between CYP1A1 genotype, mRNA expression and enzymatic activity in humans. Pharmacogenetics 4: 242-246, 1994.

11. Wang XL, Greco M, Sim AS, Duarte N, Wang J and Wilcken DE: Effect of CYP1A1 MspI polymorphism on cigarette smoking related coronary artery disease and diabetes. Atherosclerosis 162: 391-397, 2002. 
12. Taspinar M, Aydos S, Sakiragaoglu O, et al: Impact of genetic variations of the CYP1A1, GSTT1 and GSTM1 genes on the risk of coronary artery disease. DNA Cell Biol 31: 211-218, 2012.

13. Achour A, Zaag I, Gueddah L, Trimeche B, Slama FB and Zemni R: Role of CYP1A1 (T6235C) polymorphism and cigarette smoking in the development of coronary heart disease in Tunisian population. J Genet 90: 303-307, 2011.

14. Cornelis MC, El-Sohemy A and Campos H: Genetic polymorphism of CYP1A2 increases the risk of myocardial infarction J Med Genet 41: 758-762, 2004.

15. Manfredi S, Federici C, Picano E, Botto N, Rizza A and Andreassi MG: GSTM1, GSTT1 and CYP1A1 detoxification gene polymorphisms and susceptibility to smoking-related coronary artery disease: a case-only study. Mutat Res 621: 106-112, 2007.

16. Yeh CC, Sung FC, Kuo LT, Hsu WP and Chu HY: Polymorphisms of cytochrome P450 1A1, cigarette smoking and risk of coronary artery disease. Mutat Res 667: 77-81, 2009.

17. Stroup DF, Berlin JA, Morton SC, et al: Meta-analysis of observational studies in epidemiology: a proposal for reporting. Meta-analysis Of Observational Studies in Epidemiology (MOOSE) group. JAMA 283: 2008-2012, 2000.

18. Wells G, Shea B, O'Connell D, et al: The Newcastle-Ottawa Scale (NOS) for assessing the quality of nonrandomised studies in meta-analyses. http://www.ohri.ca/programs/clinical_epidemiology/oxford.asp. Accessed September 14, 2012.

19. Boivin J, Griffiths E and Venetis CA: Emotional distress in infertile women and failure of assisted reproductive technologies: meta-analysis of prospective psychosocial studies. BMJ 342: d223, 2011 .

20. Millett GA, Flores SA, Marks G, Reed JB and Herbst JH: Circumcision status and risk of HIV and sexually transmitted infections among men who have sex with men: a meta-analysis. JAMA 300: 1674-1684, 2008.

21. Rohlfs RV and Weir BS: Distributions of Hardy-Weinberg equilibrium test statistics. Genetics 180: 1609-1616, 2008.

22. Cochran WG: The combination of estimates from different experiments. Biometrics 10: 101-129, 1954.

23. Higgins JP, Thompson SG, Deeks JJ and Altman DG: Measuring inconsistency in meta-analyses. BMJ 327: 557-560, 2003.
24. DerSimonian R and Laird N: Meta-analysis in clinical trials. Control Clin Trials 7: 177-188, 1986.

25. Mantel N and Haenszel W: Statistical aspects of the analysis of data from retrospective studies of disease. J Natl Cancer Inst 22: 719-748, 1959.

26. Egger M, Davey Smith G, Schneider M and Minder C: Bias in meta-analysis detected by a simple, graphical test. BMJ 315: 629-634, 1997.

27. Jarvis MD, Palmer BR,Pilbrow AP, et al: CYP1A1 MSPI (T6235C) gene polymorphism is associated with mortality in acute coronary syndrome patients. Clin Exp Pharmacol Physiol 37: 193-198, 2010.

28. Kim SJ, Kim MG, Kim KS, Song JS, Yim SV and Chung JH: Impact of glutathione S-transferase M1 and T1 gene polymorphisms on the smoking-related coronary artery disease. J Korean Med Sci 23: 365-372, 2008.

29. Zhang XM, Xu G, Shan J, Jin GD, Huang CL and Wu KS: Association of the MspI polymorphism of cytochrome P4501A1 gene and smoking to the susceptibility to coronary artery disease.Zhonghua Xin Xue Guan Bing Za Zhi 35: 536-539, 2007 (In Chinese).

30. Nebert DW and Dalton TP: The role of cytochrome P450 enzymes in endogenous signalling pathways and environmental carcinogenesis. Nat Rev Cancer 6: 947-960, 2006.

31. Martucci CP and Fishman J: P450 enzymes of estrogen metabolism. Pharmacol Ther 57: 237-257, 1993.

32. Chen C, Huang Y, Li Y, Mao Y and Xie Y: Cytochrome P450 1A1 (CYP1A1) T3801C and A2455G polymorphisms in breast cancer risk: a meta-analysis. J Hum Genet 52: 423-435, 2007.

33. Wang JJ,Zheng Y, Sun L, et al: CYP1A1 Ile462Val polymorphism and susceptibility to lung cancer: a meta-analysis based on 32 studies. Eur J Cancer Prev 20: 445-452, 2011.

34. Huang M, Chen Q, Xiao J, Zhao X and Liu C: CYP1A1 Ile462Val is a risk factor for ovarian cancer development. Cytokine 58: 73-78, 2012.

35. Androutsopoulos VP, Tsatsakis AM and Spandidos DA: Cytochrome P450 CYP1A1: wider roles in cancer progression and prevention. BMC Cancer 9: 187, 2009.

36. Peters J and Mengersen K: Selective reporting of adjusted estimates in observational epidemiology studies: reasons and implications for meta-analyses. Eval Health Prof 31: 370-389, 2008. 